\title{
Rapid genomic changes in Drosophila melanogaster adapting to desiccation stress in an experimental evolution system
}

\author{
Lin Kang $^{1 \dagger}$, Dau Dayal Aggarwal ${ }^{2 \dagger}$, Eugenia Rashkovetsky ${ }^{2}$, Abraham B. Korol $^{2}$ and Pawel Michalak ${ }^{* *}$
}

\begin{abstract}
Background: Experimental evolution studies, coupled with whole genome resequencing and advances in bioinformatics, have become a powerful tool for exploring how populations respond to selection at the genome-wide level, complementary to genome-wide association studies (GWASs) and linkage mapping experiments as strategies to connect genotype and phenotype. In this experiment, we analyzed genomes of Drosophila melanogaster from lines evolving under long-term directional selection for increased desiccation resistance in comparison with control (no-selection) lines.

Results: We demonstrate that adaptive responses to desiccation stress have exerted extensive footprints on the genomes, manifested through a high degree of fixation of alleles in surrounding neighborhoods of eroded heterozygosity. These patterns were highly convergent across replicates, consistent with signatures of 'soft' selective sweeps, where multiple alleles present as standing genetic variation become beneficial and sweep through the replicate populations at the same time. Albeit much less frequent, we also observed line-unique sweep regions with zero or near-zero heterozygosity, consistent with classic, or 'hard', sweeps, where novel rather than pre-existing adaptive mutations may have been driven to fixation. Genes responsible for cuticle and protein deubiquitination seemed to be central to these selective sweeps. High divergence within coding sequences between selected and control lines was also reflected by significant results of the McDonald-Kreitman and Ka/Ks tests, showing that as many as 347 genes may have been under positive selection.
\end{abstract}

Conclusions: Desiccation stress, a common challenge to many organisms inhabiting dry environments, proves to be a very potent selecting factor having a big impact on genome diversity.

Keywords: Desiccation stress, Experimental evolution, Drosophila, Rapid adaptation, Selective sweep, Evolutionary genomics

\section{Background}

While desertification is expected to increase as a result of global climate change and anthropogenic influence, understanding the genomic basis of evolutionary responses to these environmental changes and associated challenges at the organismal level, such as desiccation, is imperative. Organisms are capable of surprisingly rapid adaptations to environmental challenges, such as the application of pesticides [1] or antibiotics [2], suggesting an ample supply of adaptive genetic variation and pervasive impact of positive

\footnotetext{
* Correspondence: pawel@vbi.vt.edu

${ }^{\dagger}$ Equal contributors

'Biocomplexity Institute, Virginia Tech, Blacksburg, VA 24061, USA

Full list of author information is available at the end of the article
}

directional selection. As the environment changes, alleles that previously segregated neutrally, or were only weakly influenced by selection, may become targets of strong selection, in addition to newly arising adaptive mutations. Yet, the precise mode by which beneficial mutations contribute to adaptation remains largely unclear [3].

Recent studies show that adaptations frequently result from 'soft' selective sweeps, where multiple adaptive alleles sweep through the population in parallel, either because the alleles were already present as standing genetic variation or arose independently by recurrent de novo mutations [4]. Alternatively, selective sweeps can be 'hard', where a novel beneficial allele arises after the onset of selection and sweeps through the population [5]. Whether 
adaptation originates through hard or soft sweeps depends mostly on the availability of beneficial mutations $[6,7]$, with hard sweeps taking place when beneficial alleles are absent at the onset of selection and when the waiting time for adaptive mutations to arise is long [4]. Conversely, soft sweeps are more likely to occur when the waiting time until an adaptive mutation arises is shorter than the time it takes for this mutation to spread through the population. In a hard selective sweep, all adaptive alleles coalesce into a single mutation [8], leaving characteristic footprints on neighboring genomic regions, such as a reduction in genetic diversity surrounding the adaptive site $[5,9,10]$, a distinct long haplotype $[11,12]$, and an excess of highfrequency derived alleles and singletons [13-15]. By contrast, soft sweep footprints are less pronounced and therefore more difficult to discern from surrounding neutral polymorphism, since lineages coalesce into two or more alleles, and adaptive loci may remain moderately diverse in the population $[16,17]$.

A number of approaches have been developed to detect selective sweeps in population genetic data, using a combination of methods based on LD or haplotype structure [17-21], background allele frequency spectrum from Poolseq data [22], as well as the interplay between recombination and ancestral variation [23]. Haplotype data can also be used to distinguish between signatures of soft and hard sweeps $[4,12,24]$. However, these two might not be easily separable, particularly when hard sweeps are incomplete or when recombination in distant regions linked to hard sweep sites creates patterns of polymorphism that closely mirrors what is expected to be found near soft sweeps, the so-called soft-shoulder effect [25]. These spurious soft sweeps may appear as far as kilobases or even megabases away from the true selected target under moderately strong selection [25].

Experimental evolution uses well-defined selection protocols to force phenotypic divergence, which combined with genome-wide scans ('evolve-and-resequence') may narrow down the candidate target regions under positive selection [26-30]. Experimental evolution provides a unique advantage compared to other evolutionary approaches: the ability to replicate an experiment under identical conditions, and thus to distinguish between stochastic and deterministic effects. This replication provides immediate clues about genetic parallelism that can be used to separate soft sweeps from hard sweeps, since sweep signatures parallel between replicates under the same selection pressure, either due to shared standing genetic variation or recurrent mutations, will by definition rule out hard sweeps (although replicatespecific sweep signatures will not automatically preclude soft sweeps). Parallel genetic changes have been observed in experimental evolution experiments with Escherichia coli [31-34], yeast [35], Caenorhabditis elegans [36], and Drosophila melanogaster [26]. While genetic parallelism in the first two systems was due to recurrent mutations, standing genetic variation shared between experimental replicates is the most likely explanation for evolutionary convergence in such organisms as Drosophila.

Drosophila provide an exceptional model to study the ability to survive droughts, which pose a dramatic ecological and physiological challenge to most organisms inhabiting desert and mesic environments [37]. Xericadapted Drosophila species are characterized by relatively high desiccation resistance [38, 39], lower water loss rates $[37,38]$, and decreased metabolic rates under dry conditions [40]. Artificial selection for increased desiccation resistance in $D$. melanogaster leads to critical metabolic alterations, including augmented accumulation of glycogen and differential metabolism of carbohydrates [41]. At the transcriptional level, desiccation stress in Drosophila mojavensis alters genes associated with metabolic regulation and carbohydrate metabolism [42], including cuticular hydrocarbons [43].

In this study we assessed changes in D. melanogaster genomes, with the focus on signatures of positive selection, associated with laboratory selection for increased desiccation resistance over 48 generations (Fig. 1). This experimental evolution system resulted in strong adaptive responses leading to increased desiccation resistance and changes in recombination frequencies [44]. Here we show that the selected lines also underwent extensive changes at the genomic level, with a high degree of positive selection-driven sequence divergence and zero or near-zero heterozygosity across numerous sites, producing patterns consistent with selective sweeps.

\section{Results}

\section{Polymorphism scanning}

Three desiccation lines after long-term selection and three parallel control lines (no desiccation) were Illumina-sequenced using pooled genomes per line (Pool-seq). We found a total of 1,217,242 polymorphic sites, 213,819 of which were fixed in all control lines and 603,702 were fixed in all desiccation lines. A total of 993,058 , or $81.58 \%$ of the $1,217,242$, variants were shared with the Drosophila Genetic Reference Panel lines (DGRP2 [45]). Among the fixed sites in desiccation lines, 83,875 (13.9\%) were in coding regions, compared to $28,539(13.3 \%)$ fixed sites in coding regions of control lines (Fisher exact test $P=2.67 \times 10^{-10}$ ). A total of 20,185 polymorphic sites were experiment-specific, i.e. one allele fixed in all control lines and an alternative allele fixed in all desiccation lines (Tables 1 and 2, Additional file 1). These SNPs include 745 fixed non-synonymous sites within coding sequences, significantly enriched (relative to all other SNPs in the genome; FDR $P<0.05$ ) for functions related to response to copper ion (5 genes), nuclear chromosome part (16 genes), and proteolysis (42 genes) 


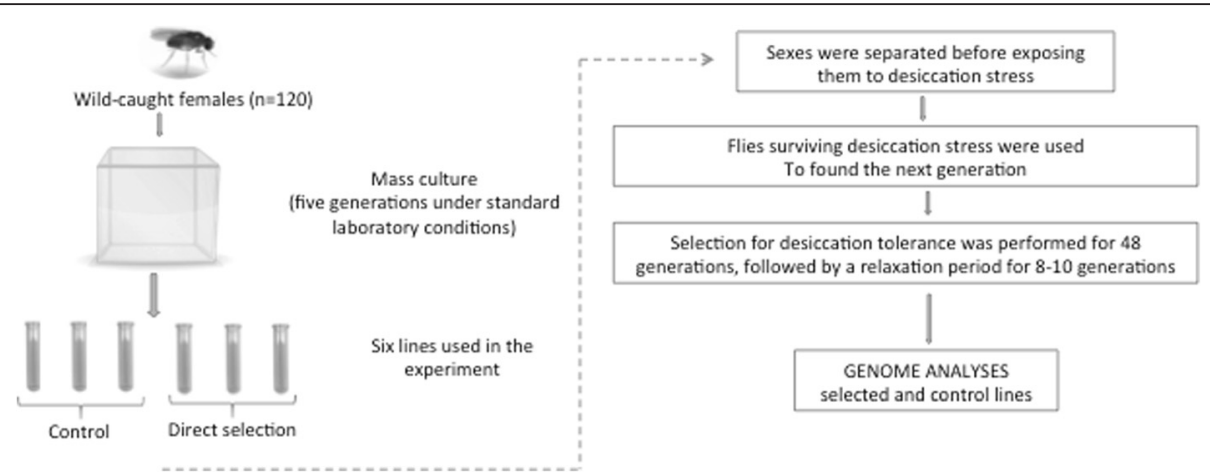

Fig. 1 Experimental setup of the experimental evolution experiment for increased desiccation resistance in Drosophila melanogaster

(Additional file 2). We found 19 non-synonymous sites with the same allele fixed in all control lines and two out of the three desiccation lines but with an alternative allele fixed in the remaining desiccation line, a pattern consistent with hard selective sweeps. Examples of these non-synonymous fixed changes occurring in only one desiccation line include chr3L:6319299G $>\mathrm{C}$ in olfactory receptor gene Or65b, with one of the selection lines, AK2-3, having allele "G" while all other five lines containing "C", which results in amino acid change from Leu to Phe. Other genes affected by such SNPs include Ubp64E, scny, QC, Mdr65, ndl, Cpr72Ec, and $a b d-A$ (see Additional file 3 for a complete list). These SNPs tend to be clustered across two islands on chromosome arm $3 \mathrm{~L}$ (permutation test $P=2.01 \times 10^{-6}$, Additional file 3 ), suggesting effects of selection.

\section{Positive selection signatures}

We used Pool-HMM based on a Hidden Markov Model (HMM) to detect possible selective sweep regions [22]. We found 118, 133, and 121 selective sweep regions in selection/desiccation lines AK2_1, AK2_2, and AK2_3, respectively, and 67 regions were shared among all three of them. By comparison, there were 136, 123, and 103 putative selective sweep regions in control lines AK2_4, AK2_7, and AK2_8, respectively, and 49 regions were shared among all three of them. Even though the sheer number of putative sweep regions was similar between the desiccation group and the control group (chi square test $P=0.144$ ), the total length of shared sweep regions among the lines in the desiccation group was 12.2 times longer than the length of shared regions in the control group (15.2 times longer on autosomes and 7.6 times longer on X chromosome, Fig. 2a), and the mean length in the desiccation group was significantly greater than that in the control group (Wilcoxon rank test $P=0.031$, Fig. 2b). Lower nucleotide diversity is expected within selective sweep regions. To test this prediction, we calculated heterozygosity in $100 \mathrm{~kb}$ windows with a step of $10 \mathrm{~kb}$. The overall heterozygosity in the desiccation group was indeed lower compared to heterozygosity of the control group $(0.12 \pm 0.11$ and $0.24 \pm 0.08$ (mean \pm SD) for autosomes in desiccation and control group, respectively; $0.11 \pm 0.11$ and $0.16 \pm 0.11$ for $\mathrm{X}$ chromosome in desiccation and control group, respectively). The average heterozygosity in shared sweep regions in the desiccation group was 0.02 . These results are consistent with a strong selection pressure in the desiccation group, and the lower heterozygosity in $\mathrm{X}$ chromosome likely reflects the difference in effective population sizes between $\mathrm{X}$ chromosome and autosomes. Simulations of neutral evolution under conditions similar to our experimental setup, based on genomic variation of DGRP2, failed to produce regions of decreased heterozygosity comparable

Table 1 Distribution of SNPS

\begin{tabular}{llclc}
\hline Categories & \# of SNPs & \% of SNPs & Sequencing coverage & Density of SNP (per Kb) \\
\hline Intergenic & 376,955 & 30.97 & $33,640,807$ & 11.21 \\
5'-UTR & 32,099 & 2.64 & $4,011,958$ & 8.00 \\
CDS (synonymous) & 121,477 & 9.98 & $22,691,151$ & 7.36 \\
CDS (non-synonymous) & 45,577 & 3.74 & & 11.13 \\
Intron & 578,685 & 47.54 & $51,989,650$ & 7.71 \\
3'-UTR & 50,850 & 4.18 & $6,599,093$ & 10.19 \\
ncRNA & 11,599 & 0.95 & $1,138,125$ & 10.14 \\
Total: & $1,217,242$ & 100.00 & $120,070,784$ & \\
\hline
\end{tabular}


Table 2 Distribution of fixed SNPs in both control group and desiccation group

\begin{tabular}{llcll}
\hline Categories & \# of SNPs & \% of SNPs & Sequencing coverage & Density of SNP (per Kb) \\
\hline intergenic & 6,808 & 33.73 & $33,640,807$ & 0.20 \\
5'-UTR & 533 & 2.64 & $4,011,958$ & 0.13 \\
CDS (synonymous) & 2,056 & 10.19 & $22,691,151$ & 0.12 \\
CDS (non-synonymous) & 745 & 3.69 & & 0.18 \\
Intron & 9,108 & 45.12 & $51,989,650$ & 0.11 \\
3'-UTR & 755 & 3.74 & $6,599,093$ & 0.16 \\
ncRNA & 181 & 0.90 & $1,138,125$ & $120,070,784$ \\
Total: & 20,186 & 100.00 & & 0.17 \\
\hline
\end{tabular}

with experimental ones (Additional file 4). Simulated drift effects were also unable to drive the same allele to fixation in three lines while the alternative allele was fixed in the other three lines (there were 745 such nonsynonymous sites in the experimental data). When selection was added to the simulations, we were able to generate large genomic regions $(\sim 2 \mathrm{Mb})$ of depleted heterozygosity comparable to observed sweep regions, but only when the selected allele was initially present at a very low frequency $(\sim 1 / 400)$ and assigned high relative fitness ( 4) (Additional file 4).
We found blocks of extremely low heterozygosity overlapping with the HMM-identified sweep regions in the desiccation group (Fig. 3). Mean heterozygosity values in non-sweep regions and sweep regions for control lines were 0.238 and 0.144 , respectively, and 0.184 and 0.036 for selection lines, respectively. As expected, the reduction in heterozygosity between sweep regions and non-sweep regions was thus stronger in selection lines (Wilcoxon rank sum test $P=0.016$ ). Tajima's $D$ and $\pi$ values produced a very similar pattern to heterozygosity along the sweep regions (Fig. 3, Additional file 5). Accordingly,

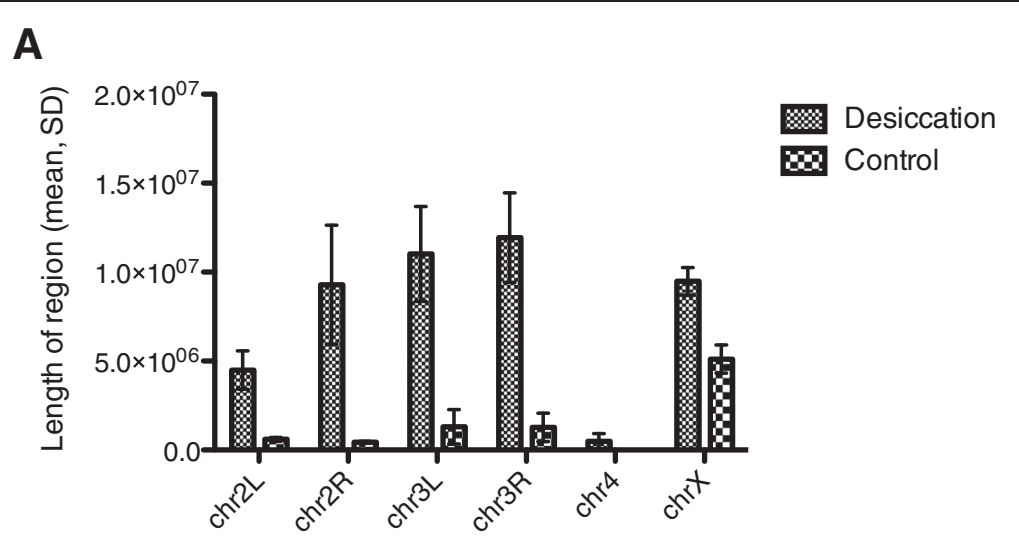

B

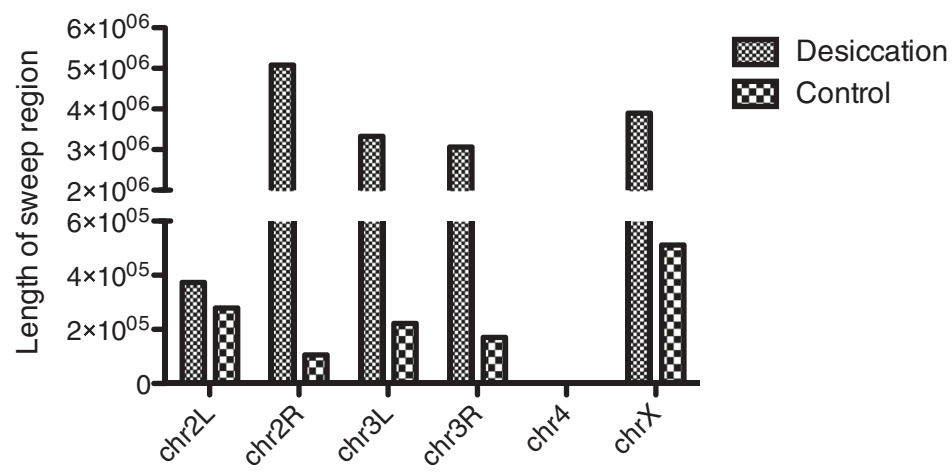

Fig. 2 Sweeps found in the Desiccation group and the Control group. Average length of sweep regions (a) and length of shared sweep regions (b) for Desiccation and Control groups by chromosomes 


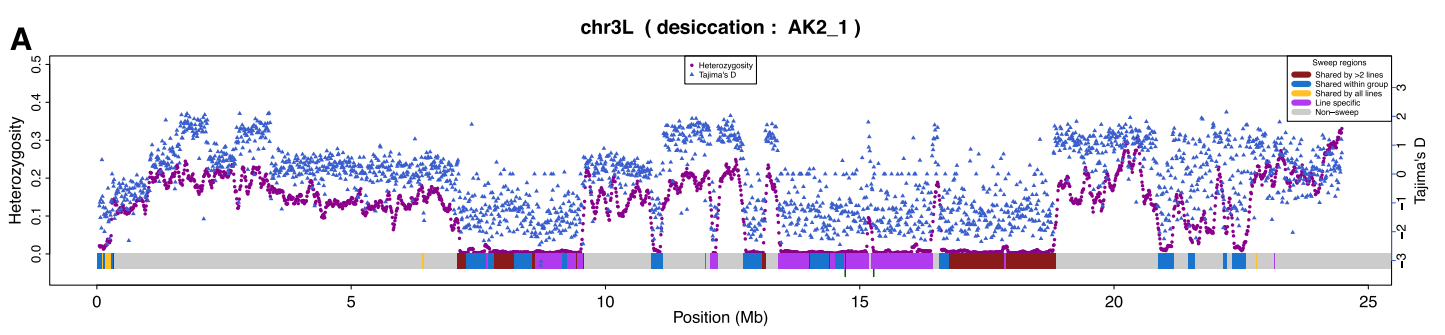

chr3L (desiccation : AK2_2)

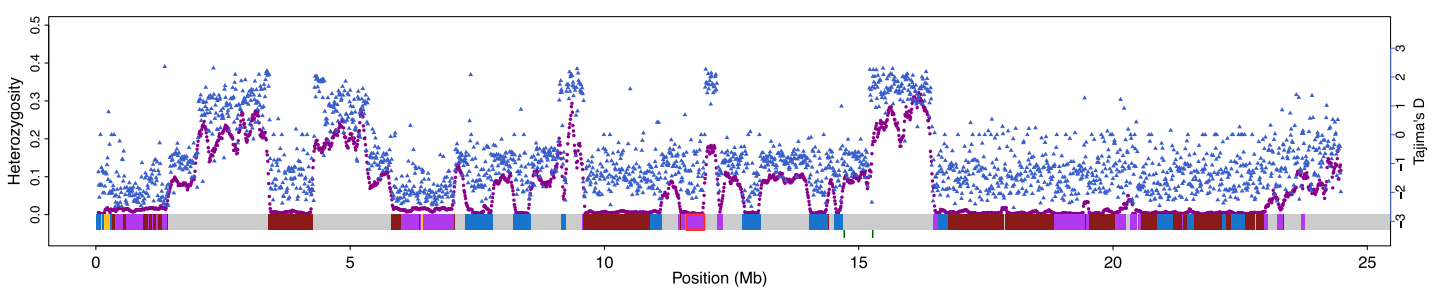

chr3L (desiccation : AK2_3)

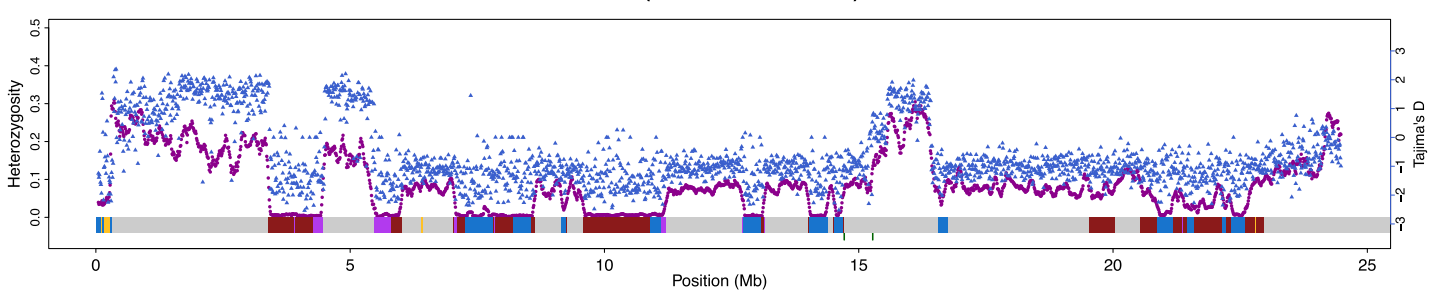

B

chr3L ( control : AK2_4)

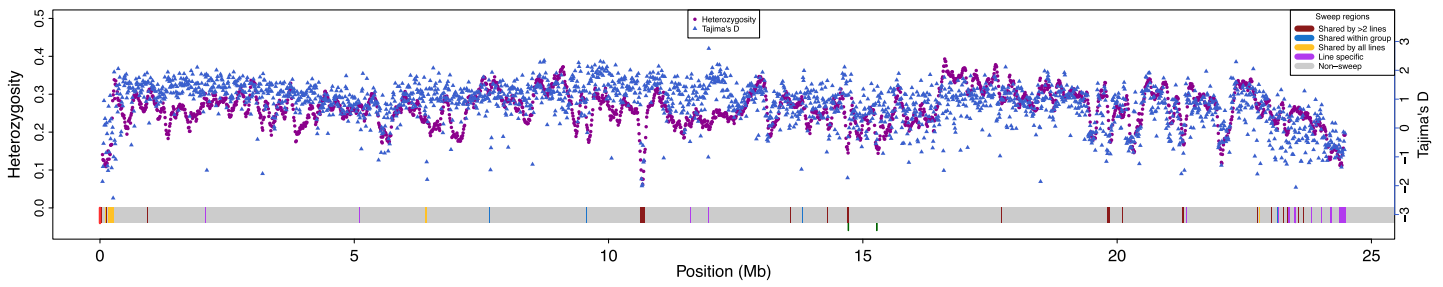

chr3L (control : AK2_7)

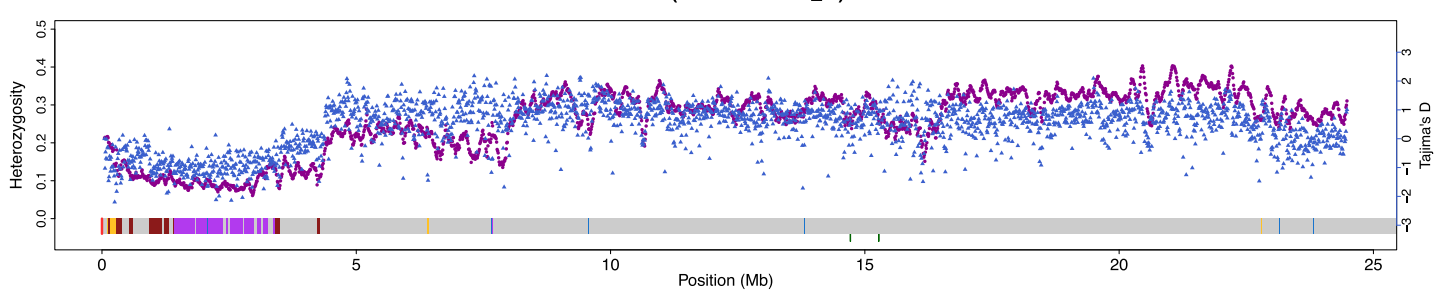

chr3L ( control : AK2_8)

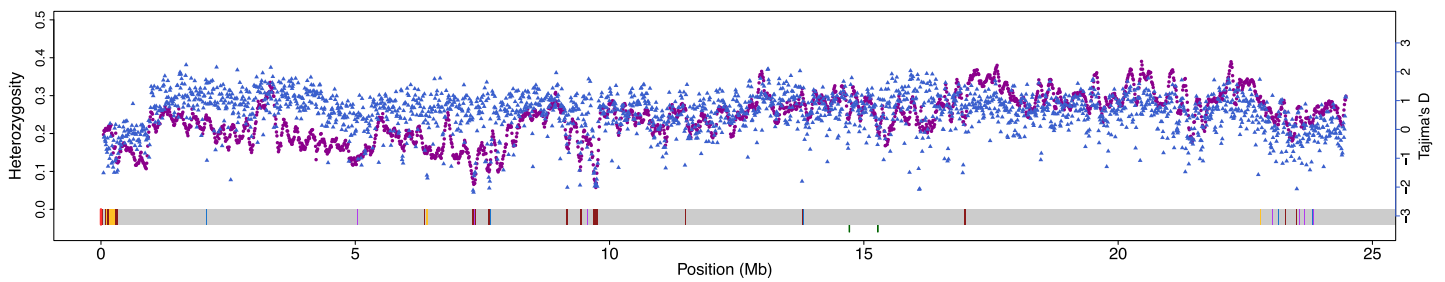

Fig. 3 (See legend on next page.) 
(See figure on previous page.)

Fig. 3 Heterozygosity and Tajima's D values plotted against the putative selective sweep signatures (horizontal color blocks) along chromosomal arm $3 \mathrm{~L}$ in a three D. melanogaster desiccation lines (AK2_1, AK2_2, AK2_3), and $\mathbf{b}$ the negative control lines (AK2_4, AK2_7, AK2_8). Horizontal color blocks correspond to putative sweep regions: line-specific (red), experimental group -specific (blue), shared by all groups (yellow), and no sweep (grey)

sweep signatures shared by all lines in the desiccation group likely represent soft sweeps, since the parallelism is most easily explained by preexisting variation at a sufficiently high population frequency to not be stochastically lost by drift. We found a total of 67 putative soft sweep regions (Additional file 6). GO enrichment analysis of genes involved in the shared sweep regions in the desiccation group shows functional enrichments for organophosphate ester transmembrane transporter activity, electron carrier activity, water channel activity, and proteolysis (Additional file 7). On the other hand, line-specific sweep signatures (purple blocks in Fig. 3 and Additional file 5) that are not adjacent to other shared sweep regions and have extremely low heterozygosity values are possible hallmark signatures of hard sweeps based on new mutations. We found five hard sweep candidates (average heterozygosity of 0.007 , Fig. 3, Additional files 5 and 8) that contain 435 genes, with enrichment of $\mathrm{GO}$ terms related to acetaldehyde dehydrogenase (acetylating) activity, organic cation transmembrane transporter activity, active transmembrane transporter activity, and a variety of metabolic processes (Additional file 9). Similar enrichment patterns were also found in a desiccation resistance study of differentiation expression genes in Drosophila mojavensis [42]. A total of 178 genes from the sweep regions shared by our desiccation lines were listed by Rajpurohit et al. [43] as genes differentially expressed in $D$. mojavensis under desiccation stress (Additional File 10).

All of the 19 non-synonymous sites with the same allele fixed in all control lines and two out of the three desiccation lines, but with an alternative allele fixed in the remaining desiccation line, fell within the HMMidentified sweep regions. Seven of the SNPs unique for a single desiccation lines were also located in a sweep region reported only in that line (three genes on chromosome arm $3 \mathrm{~L}, U b p 64 E$, scny, and $C p r 72 E c$, and one gene on $3 \mathrm{R}, a b d-A$ ), consistent with a hard sweep pattern. Three of the 19 SNPs from one block (chr2R), containing genes CG30391, CG30393 and CG42672, were located in putative sweep regions shared by all three desiccation lines.

\section{Positive selection tests with MKT and Ka/Ks}

Using the McDonald-Kreitman test (MKT), we found 15 genes likely under positive selection $(\operatorname{DoS}>0, P<0.05)$ and 9 genes under negative selection $(\operatorname{DoS}<0, P<0.05)$ when control and desiccation groups were compared (Table 3). Positively selected genes include Mucin $68 \mathrm{Ca}$, an extracellular matrix structural constituent [46], characterized by 11 fixed non-synonymous substitutions between control and desiccation groups. Another gene under positive selection, foraging (for), is involved in functions related to sucrose response [47], regulation of heart contraction [48], and feeding behavior [49, 50]. Ninjurin $A$, involved in cell adhesion [51] and tissue regeneration [52], is an example of a gene likely under negative selection. Eight of these 15 genes fell in soft sweep regions (Table 3) but none of them in hard sweep regions (see Additional file 10 for the complete list). We also calculated the $\mathrm{Ka} / \mathrm{Ks}$ ratios using fixed genotypes in each group, and found 347 genes with $\mathrm{Ka} / \mathrm{Ks}$ ratio

Table 3 MKT between control group and desiccation group

\begin{tabular}{|c|c|c|c|c|c|c|}
\hline \#GENEID & DS & DN & PS & PN & Dos & $p$-value (Fisher) \\
\hline CG2211 & 2 & 2 & 22 & 0 & 0.5 & 0.0185 \\
\hline CG31038 & 0 & 2 & 22 & 4 & 0.8462 & 0.0397 \\
\hline$C G 7213^{a}$ & 1 & 3 & 14 & 2 & 0.625 & 0.032 \\
\hline CG8545 & 0 & 4 & 17 & 9 & 0.6538 & 0.0261 \\
\hline CG8785 & 0 & 3 & 18 & 2 & 0.9 & 0.0056 \\
\hline CG9304 & 0 & 4 & 27 & 10 & 0.7297 & 0.0099 \\
\hline Cngl & 1 & 2 & 16 & 1 & 0.6078 & 0.0456 \\
\hline GM130 & 2 & 4 & 25 & 7 & 0.4479 & 0.0466 \\
\hline $\operatorname{ir} 7 d^{a, b}$ & 1 & 4 & 20 & 6 & 0.5692 & 0.0274 \\
\hline Muc68Cab & 0 & 11 & 29 & 55 & 0.3452 & 0.0166 \\
\hline$b t s z^{a, b}$ & 1 & 4 & 75 & 26 & 0.5426 & 0.022 \\
\hline for ${ }^{b}$ & 1 & 4 & 24 & 0 & 0.8 & 0.0002 \\
\hline hay & 3 & 2 & 21 & 0 & 0.4 & 0.0308 \\
\hline mnd & 1 & 2 & 14 & 0 & 0.6667 & 0.0221 \\
\hline $\operatorname{sog}^{b}$ & 0 & 2 & 20 & 0 & 1 & 0.0043 \\
\hline CG10170 & 4 & 1 & 24 & 51 & -0.48 & 0.0484 \\
\hline CG13540 & 2 & 0 & 0 & 8 & -1 & 0.0222 \\
\hline CG15373 & 4 & 1 & 0 & 4 & -0.8 & 0.0476 \\
\hline CG15394 & 2 & 0 & 0 & 7 & -1 & 0.0278 \\
\hline CG17734 & 3 & 0 & 0 & 5 & -1 & 0.0179 \\
\hline CG43798 & 2 & 0 & 2 & 16 & -0.8889 & 0.0316 \\
\hline Caf1-180 & 14 & 3 & 18 & 18 & -0.3235 & 0.0355 \\
\hline NijA & 11 & 2 & 0 & 3 & -0.8462 & 0.0179 \\
\hline hang & 15 & 0 & 6 & 3 & -0.3333 & 0.0415 \\
\hline
\end{tabular}

$D S$ synonymous substitution between populations, $D N$ non-synonymous substitution between populations, $P S$ synonymous polymorphisms within populations, $P N$ non-synonymous polymorphisms within populations afalls in putative soft sweep regions

${ }^{\mathrm{b}}$ with $\mathrm{Ka} / \mathrm{Ks}$ ratio greater than 1 
greater than 1 (Additional file 10). Among these genes, 277 (79.8 \%) showed one fixed non-synonymous substitution- but no fixed synonymous substitution-difference between desiccation and control group. A total of 9 out of 15 genes with positive selection of MKT also had $\mathrm{Ka} / \mathrm{Ks}$ greater than 1 (Table 3). The 347 genes with $\mathrm{Ka} / \mathrm{Ks}$ greater than 1 showed a significant enrichment of GO terms related to gastrulation and detection of chemical stimulus (Additional file 11). Although immediate relevance of these GO terms to desiccation is unclear, it is likely that pleiotropic activities of genes involved are more directly related to desiccation. For example, genes belonging to the "gastrulation" GO, such as sog (short gastrulation), are not only responsible for amnion and serosa development, but also contribute to ectoderm and cuticle formation, with plausible implications for desiccation tolerance [53]. Overall, these results show that selection for desiccation resistance is a very potent evolutionary force leading to adaptive differentiation. To summarize this differentiation, we computed per-site $\mathrm{F}_{\mathrm{ST}}$ values based on $>1$ million SNPs. Average pairwise $\mathrm{F}_{\mathrm{ST}}$ values were 0.081 and 0.110 among selected and control lines, respectively, compared to 0.242 - the average $\mathrm{F}_{\mathrm{ST}}$ for all selection-control line combinations.

\section{Discussion}

We notice that genomic signatures of adaptive responses to directional selection for increased desiccation resistance are highly convergent among selected lines, a pattern consistent with soft selective sweeps operating on standing genetic variation, similar to that found by others [26, 28, 54, 55]. However, unlike Burke et al. [26], who analyzed genomes of $D$. melanogaster selected for accelerated development for over 600 generations, we found numerous sites brought to fixation in the selected lines, including a number of line-specific fixations with signatures of classic, or hard, sweeps in the immediate vicinity. In the selected lines, fixed non-synonymous substitutions shared by all three selected lines were about 40 times more abundant than fixed non-synonymous substitutions limited to only one selected line, and line-convergent signatures of selective sweeps were 13 times more abundant than line-specific selective sweep signatures. These estimates confirm that albeit soft sweeps are expected to leave more subtle footprints on population genomic data compared with hard sweeps, the former actually emerges as the dominant mode of rapid adaptations in Drosophila $[4,26]$. These results also suggest that availability of beneficial mutations for rapid adaptations is not a severely limiting factor in $D$. melanogaster. Conversely, hard sweeps are predicted to be dominant only: (i) in consistently small populations; (ii) when adaptation is driven by weak selection in populations of significantly fluctuating size; or (iii) when the adaptive mutation rate is very low, such as when only a specific combination of mutations is adaptive whereas individual mutations are not [4].

We singled out a number of line-specific sweep regions with extremely low heterozygosity or Tajima's D values, and high fixation rate of the line-unique alleles, including non-synonymous substitutions, as potential hard sweep examples. However, rather than having originated from new beneficial mutations, these putative hard sweep regions, as confirmed by our simulations (Additional file 4), may have actually started from preexisting low-frequency variants unique to a single haplotype background [56]. Under such a scenario the spread and subsequent fixation of the beneficial haplotype could have led to the observed characteristic loss of heterozygosity in a single population, while the beneficial variant might have been lost in the remaining populations due to drift at the low initial frequency, thus resulting in genomic signatures of hard sweeps without the necessity for selection on new mutations. In particular, three genes from chromosome arm $3 \mathrm{~L}$ were affected by these sweeps: Ubp64E, scny, and $C p r 72 E c$. Interestingly, both $U b p 64 E$ and scny are responsible for protein deubiquitination and stabilization $[57,58]$, whereas both scny and Cpr $72 E c$ contribute to the structure, growth, and molting of chitin-based cuticle $[59,60]$. The ubiquitin-mediated protein degradation has been known to play an important role in desiccation resistance in plants [61] and nematodes [62], while cuticle is instrumental as a barrier against desiccation in terrestrial arthropods, including insects [63]. However, pinpointing true targets of selection, especially in soft sweeps, is very difficult without information about ancestral alleles. We tested Gene Ontology enrichments in the putative sweep regions, but these results only unlikely do represent more than LD effects, or 'innocent bystanders' [64], especially in the presence of 'soft shoulders' originating from hard sweeps [25].

Similar to our study, but using hypoxic stress for directional selection, Zhou et al. [65] reported significant enrichment for fixed SNPs and fairly large genomic regions to have been affected by adaptive responses to the selection regime. They identified 188 candidate genes, eight of which were previously implicated in hypoxia or similar phenotypes and 12 were linked to the Notch pathway. An even more dramatic response to experimental evolution with a high rate of convergent fixation of preexisting alleles was recorded in C. elegans [36]. These worm populations were founded with a fixed pair of temperature-sensitive deleterious mutations introgressed into multiple wild genetic backgrounds and allowed to evolve for 50 generations with a mixed mating system. Near-complete fixation, rather than moderate changes in allele frequencies, occurred throughout almost the entire genome in these populations, and 
entire chromosomal segments became fixed due to strong selection and low effective recombination rates [36].

A selective sweep generates an effectively linked region around the sweeping site, and the region size grows with the strength of positive selection and shrinks with recombination rate [9]. The mode and intensity of selection might also explain why adaptive alleles are driven to fixation in some experimental evolution experiments with D. melanogaster ([65] and this study) but not in others $[26,54,66]$. Allele frequencies may plateau without becoming fixed due to heterozygote overdominance or selection on a complex trait with several contributing loci, in which case adaptive allele trajectories are expected to rapidly incline as long as the trait is far away from the fitness optimum, but taper off as the optimum is being approached $[67,68]$.

Given the fact that we also recorded signatures of selective sweeps in the negative control lines, even though at least 12 times less extensive than in experimental lines, suggests a high rate of false discovery rate, experimental noise or inadvertent laboratory selection. Alternatively, long-range LD in our experimental populations could have arisen as a byproduct of the founders being a small sample of the much larger population, leading to a sampling error and spurious correlations between sites separated by large distances [69]. However, observed at the same time pervasive convergence among experimental replicates suggests that sampling error is likely negligible.

\section{Conclusions}

These results demonstrate the utility of the experimental evolution approach in exploring how patterns of genomic differentiation are shaped by selection for stress resistance. Desiccation, the equilibration of an organism to the relative humidity of the surrounding atmosphere, is an intense stress factor leading to rapid adaptive responses in Drosophila under experimental evolution settings. Experimental lines of $D$. melanogaster subject to desiccation stress over 48 generations exhibited rapid resistance increase, accompanied by a high fixation rate and genetically parallel signatures of positive selection in coding sequences, consistent with soft selective sweep patterns.

\section{Methods}

\section{Flies and the experimental setup}

Wild individuals of $D$. melanogaster $(n=120)$ were collected in March 2009 from Madhya Pradesh, Jabalpur, India $\left(23^{\circ} 30^{\prime} \mathrm{N}\right.$; $80^{\circ} 01^{\prime} \mathrm{E}$; alt. $\left.393 \mathrm{~m}\right)$. Before the start of the selection experiment, mass culture was maintained for 5 generations under standard laboratory conditions at low density (on yeast-cornmeal-agar medium at $21{ }^{\circ} \mathrm{C}$, and $\sim 70 \%$ relative humidity) to eliminate environmental effects. For laboratory selection, virgin flies were sexed under $\mathrm{CO}_{2}$ anesthesia at least $48 \mathrm{~h}$ prior to the experiment.
Then virgin flies (3-4 days old) were placed in groups of 25 into plastic vials containing $2 \mathrm{~g}$ of silica gel and covered with discs of foam pieces. Experiments were conducted for males and females separately, since sex has been a factor known to affect desiccation resistance [70, 71]. Flies were subjected to desiccation stress until $\sim \mathrm{LT}_{70}-\mathrm{LT}_{85}$ level of mortality was reached. Control groups were processed in the same manner, excluding desiccation stress. In each generation, we examined 1000 virgin flies of each sex per replicate, of which at least 100 males and 100 females survived the $\mathrm{LT}_{70-85}$ cut-off to become the parents of the next generation. For each group (selection and control), survivors were randomly allocated into three sub-groups (three replicates). The same protocol was recapitulated for 48 generations (each next generation was subjected to analogous treatment), and then selection was relaxed for 8-10 generations before initiating the recombination tests. The control lines were not subjected to any treatment and were maintained in comparable densities to the selection lines on standard media. The influence of starvation stress on mortality rate in the desiccated groups was considered non-significant: in preliminary experiments, flies from the control groups began to die from starvation when all flies from the experimental groups (subjected to combined desiccation + starvation stress) were already dead. Desiccation resistance was measured as the time to lethal dehydration effect under dry air. Groups of 10 female/male flies were placed in dry plastic vials with $2 \mathrm{~g}$ of silica gel at the bottom and covered with discs of foam pieces. These vials were then placed in a desiccator chamber. The number of immobile individuals was counted every hour and times to lethal desiccation effects $\left(\mathrm{LT}_{100}\right)$ were recorded. In the present study, we used 3 control and 3 desiccation-resistant lines for sequencing. Average desiccation tolerance of the initial population was $14.8 \mathrm{~h}$ and $23.2 \mathrm{~h}$ (with $\mathrm{SD}=2.88$ and 3.44 ), for males and females, respectively. After 48 generations of selection, these tolerance characteristics increased to $25.3 \mathrm{~h}$ and $43.6 \mathrm{~h}$ for males and females, respectively, i.e. 3.65SDs and 5.93SDs compared to the starting population.

\section{Sequencing}

In the $F_{1}$ progeny also, we collected 15-20 virgin females and each virgin female was kept into separate small vials together with single brother. The outcome progeny, $n=100$ females was used for sequencing. Because the $F_{1}$ mothers were reared in separate vials, we achieved our target progeny ( $n=100$ females), mostly from 3-5 $F_{1}$ mother females only. TruSeq $2 \times 100$ bp libraries were constructed, barcoded, and sequenced within one lane at 31-38x coverage using Illumina’s HiSeq 2500 (Additional file 12).

\section{Mapping and genotyping}

D. melanogaster assembly ( $\mathrm{dm} 3$ ) and corresponding annotations (RefSeq) were downloaded from UCSC (http:// 
genome.ucsc.edu/) and used as reference. Raw reads were undergone quality control and filtered through FastqMcf [72]. Clean reads were then mapped to reference using BWA [73] with default parameters. For each line, genotypes were generated using GATK [74] with default parameters except for using '-sample_ploidy' for pooled data and setting heterozygosity (-heterozygosity) to 0.01 . Only sites with genotyping quality greater than 30 and minimal depth 10 and maximal depth 250 were kept. Genotypes with more than 2 alleles were discarded from further analyses. For each genotype, percentage of reference allele greater than $99 \%$ or less than $1 \%$ was considered to be homozygous or fixed genotype.

\section{$F_{S T}, \pi, \theta$, Tajima's $D$, heterozygosity}

Samtools [75] was used to generate the pileup result. SNPs within $10 \mathrm{bp}$ of indel were discarded and $\mathrm{F}_{\mathrm{ST}}$ value for each SNP was generated by Poopolation2 [77]. PoPoolation [77] was used to estimate $\pi$, Watterson's $\theta$ and Tajima's D with the window size set to $10 \mathrm{~Kb}$. Heterozygosity was calculated based on $100 \mathrm{~Kb}$ sliding window with a step of $10 \mathrm{~Kb}$.

\section{Sweep region identification}

Sweeps were identified by Pool-hmm [22], a hidden Markov model for detecting selective sweep based on Pool-Seq data. The parameters used in Pool-hmm were set to be "-n 100 -c 5 -C 400 -q 20 -e sanger -p $-\mathrm{k} 0.0000000001$ ", and "-theta" was set to be the $\theta$ estimated for each sample.

\section{Neutrality and positive selection simulations}

Genome-wide neutrality simulations were performed using forqs [78] to test whether observed patterns of genomic differentiation could result from drift alone. The haplotype data for the simulation under a neutrality model were derived from the Drosophila Genetic Reference Panel 2 (DGRP2, http://dgrp2.gnets.ncsu.edu/) containing 205 inbreed lines [45]. Missing genotypes were set as heterozygous. The mass-breeding phase was simulated for 1,000 generations with a population size of 100,000 . Then conditions mimicking our experimental study were simulated with an initial population size of 120 , followed by 5 generations of mass culture (population size 2,000), 48 generations of analogous selection (population size 200), 10 generations of analogous relaxed selection, and the final generation with the population size of 100. In total, 6 chromosomes/arms (chr2L, chr2R, chr3L, chr3R, chr4 and chrX) were simulated, and the recombination rate was set to 2. Simulations were repeated several times to mimic the replicas in our study. Heterozygosity was calculated based on the same window size as for the real experimental data. Selection simulations involving a single site on chromosome $2 \mathrm{~L}$ were also performed. We used a value of 0.2 as relative fitness ('FitnessFunction') for 'genotype 0' to represent the $\sim$ LT70-LT85 level of mortality in our experimental desiccation lines. The mass-bred population from the neutrality simulation was used as the initial population (size of 200) and a total of 48 generations were simulated. A range of parameters for fitness and initial allele frequency were tested and each parameter-combination was repeated 10 times (Additional file 4).

\section{GO Enrichment}

To take the gene length into account, an unbiased analysis of gene set enrichment using GOWINDA [79] was performed by considering only SNPs in the CDS regions. Gene mode was used (-mode, assumes all SNPs within a gene are in linkage disequilibrium) with parameters of $1,000,000$ simulation (-simulations), the minimum gene number in GO category of 2 (-min-genes) and all SNPs as the background (-snp-file). Enrichment analysis for genes with $\mathrm{Ka} / \mathrm{Ks}$ greater than 1 was performed on DAVID site (http://david.abcc.ncifcrf.gov/).

\section{McDonald-Kreitman test and $\mathrm{Ka} / \mathrm{Ks}$ ratio}

Even though McDonald-Kreitman (MK) test was originally designed for testing between species, we use the test in this study to assess adaptive divergence between groups. MK test compares the number of synonymous (Ds) and non-synonymous (Dn) substitutions between species with the number of synonymous (Ps) and nonsynonymous $(\mathrm{Pn})$ polymorphisms within species [80]. $P$-values were computed using Fisher exact test. The $\mathrm{Ka} / \mathrm{Ks}$ ratio, which is the number of non-synonymous substitutions per non-synonymous site $(\mathrm{Ka})$ to the number of synonymous substitutions per synonymous site (Ks), was calculated according to Nei's approximate method [81].

\section{Availability of supporting data}

The sequence data supporting the results of this article are available in the NCBI's Sequence Read Archive (SRA) under the accession number SRP066877.

\section{Additional files}

Additional file 1: Table listing the genotype fixed in both control group and desiccation group. (XLSX $2892 \mathrm{~kb}$ )

Additional file 2: Table listing the enrichment analysis of fixed nonsynonymous polymorphic sites. (XLSX $45 \mathrm{~kb}$ )

Additional file 3: Table listing the putative new mutations in desiccation lines. (XLSX $44 \mathrm{~kb}$ )

Additional file 4: Figures illustrating neutrality-selection simulation results. (A-F) Heterozygosity values along chromosomes from simulated neutral evolution. (G-H) Average sizes of regions with low heterozygosity values for different parameter combinations under a selection simulation. (I) Examples of heterozygosity values along chromosome $2 \mathrm{~L}$ from a selection simulation. (PDF $541 \mathrm{~kb}$ ) 
Additional file 5: Figures of heterozygosity and Tajima's D values plotted against the putative selective sweep signatures (horizontal color blocks) along chromosomal 2 L, 2R, 3R, and X. (PDF $2138 \mathrm{~kb}$ )

Additional file 6: Table listing the regions of putative soft selection sweeps. (XLSX $41 \mathrm{~kb}$ )

Additional file 7: Table listing the enrichment analysis of genes in putative soft sweeps. (XLSX $72 \mathrm{~kb}$ )

Additional file 8: Table listing the regions of putative hard selection sweeps. (XLSX $32 \mathrm{~kb}$ )

Additional file 9: Table listing the enrichment analysis of genes in putative hard sweeps. (XLSX $41 \mathrm{~kb}$ )

Additional file 10: Table listing the MKT and $\mathrm{Ka} / \mathrm{Ks}$ for all genes. (XLSX $997 \mathrm{~kb}$ )

Additional file 11: Table listing the enrichment analysis of genes with $\mathrm{Ka} / \mathrm{Ks}$ ratio greater than 1. (XLSX $36 \mathrm{~kb}$ )

Additional file 12: Table listing the statistics of clean sequencing reads. (XLSX $35 \mathrm{~kb})$

\section{Abbreviations}

DGRP2: Drosophila Genetic Reference Panel 2; Dn: the number of nonsynonymous substitutions between species; DoS: direction of selection; Ds: the number of synonymous substitutions between species; FDR: false discovery rate; Ka: the number of non-synonymous substitutions per nonsynonymous site; Ks: the number of synonymous substitutions per synonymous site; MKT: McDonald-Kreitman test; Pn: the number of non-synonymous polymorphisms; Ps: the number of synonymous polymorphisms.

\section{Competing interests}

The authors declare that they have no competing interests.

\section{Authors' contributions}

LK analyzed the sequence data and contributed to writing, DDA conducted the experiment, ER conducted the experiment, ABK conceived the experiment and contributed to writing, and PM wrote the manuscript and contributed to the analysis. All authors have read and approved of the final manuscript.

\section{Acknowledgements}

The authors would like to thank two anonymous reviewers for their constructive criticism. The study was supported by Binational USA-Israel Science Foundation (grant BSF \# 2011438)

\section{Author details}

'Biocomplexity Institute, Virginia Tech, Blacksburg, VA 24061, USA. ${ }^{2}$ Institute of Evolution, University of Haifa, Abba Khoushy Ave 199, Haifa, Israel.

\section{Received: 8 December 2015 Accepted: 29 February 2016}

Published online: 15 March 2016

\section{References}

1. Daborn P, Boundy S, Yen J, Pittendrigh B, ffrench-Constant R. DDT resistance in Drosophila correlates with Cyp6g1 over-expression and confers cross-resistance to the neonicotinoid imidacloprid. Mol Genet Genomics. 2001:266(4):556-63.

2. Laehnemann D, Pena-Miller R, Rosenstiel P, Beardmore R, Jansen G, Schulenburg $H$. Genomics of rapid adaptation to antibiotics: convergent evolution and scalable sequence amplification. Genome Biol Evol. 2014;6(6):1287-301.

3. Peter BM, Huerta-Sanchez E, Nielsen R. Distinguishing between selective sweeps from standing variation and from a de novo mutation. PLoS Genet. 2012;8(10):e1003011.

4. Messer PW, Petrov DA. Population genomics of rapid adaptation by soft selective sweeps. Trends Ecol Evol. 2013;28(11):659-69.

5. Smith JM, Haigh J. The hitch-hiking effect of a favourable gene. Genet Res. 1974;23(1):23-35

6. Hermisson J, Pennings PS. Soft sweeps: molecular population genetics of adaptation from standing genetic variation. Genetics. 2005;169(4):2335-52.

7. Barton N. Understanding adaptation in large populations. PLoS Genet. 2010; 6(6):e1000987
8. Berg JJ, Coop G. A Coalescent Model for a Sweep of a Unique Standing Variant. Genetics. 2015;201(2):707-25.

9. Kaplan NL, Hudson RR, Langley CH. The "hitchhiking effect" revisited. Genetics. 1989:123(4):887-99.

10. Kim Y, Stephan W. Detecting a local signature of genetic hitchhiking along a recombining chromosome. Genetics. 2002;160(2):765-77.

11. Hudson RR, Bailey K, Skarecky D, Kwiatowski J, Ayala FJ. Evidence for positive selection in the superoxide dismutase (Sod) region of Drosophila melanogaster. Genetics. 1994;136(4):1329-40.

12. Garud NR, Messer PW, Buzbas EO, Petrov DA. Recent selective sweeps in North American Drosophila melanogaster show signatures of soft sweeps. PLoS Genet. 2015;11(2):e1005004.

13. Fay JC, Wu Cl. Hitchhiking under positive Darwinian selection. Genetics. 2000;155(3):1405-13.

14. Slatkin M, Hudson RR. Pairwise comparisons of mitochondrial DNA sequences in stable and exponentially growing populations. Genetics. 1991;129(2):555-62.

15. Barton NH. The effect of hitch-hiking on neutral genealogies. Genet Res. 1998;72(2):123-33

16. Przeworski M, Coop G, Wall JD. The signature of positive selection on standing genetic variation. Evolution. 2005;59(11):2312-23.

17. Pennings PS, Hermisson J. Soft sweeps III: the signature of positive selection from recurrent mutation. PLoS Genet. 2006;2(12):e186.

18. Sabeti PC, Reich DE, Higgins JM, Levine HZ, Richter DJ, Schaffner SF, et al. Detecting recent positive selection in the human genome from haplotype structure. Nature. 2002;419(6909):832-7.

19. Voight BF, Kudaravalli S, Wen X, Pritchard JK. A map of recent positive selection in the human genome. PLoS Biol. 2006;4(3):e72.

20. Zhang C, Bailey DK, Awad T, Liu G, Xing G, Cao M, et al. A whole genome long-range haplotype (WGLRH) test for detecting imprints of positive selection in human populations. Bioinformatics. 2006;22(17):2122-8.

21. Sabeti PC, Varilly P, Fry B, Lohmueller J, Hostetter E, Cotsapas C, et al. Genome-wide detection and characterization of positive selection in human populations. Nature. 2007:449(7164):913-8

22. Boitard S, Kofler R, Francoise P, Robelin D, Schlotterer C, Futschik A. Poolhmm: a Python program for estimating the allele frequency spectrum and detecting selective sweeps from next generation sequencing of pooled samples. Mol Ecol Resour. 2013;13(2):337-40.

23. Pritchard JK, Pickrell JK, Coop G. The genetics of human adaptation: hard sweeps, soft sweeps, and polygenic adaptation. Curr Biol. 2010;20(4):R208-215.

24. Messer PW, Neher RA. Estimating the strength of selective sweeps from deep population diversity data. Genetics. 2012;191(2):593-605.

25. Schrider DR, Mendes FK, Hahn MW, Kern AD. Soft shoulders ahead: spurious signatures of soft and partial selective sweeps result from linked hard sweeps. Genetics. 2015;200(1):267-84.

26. Burke MK, Dunham JP, Shahrestani P, Thornton KR, Rose MR, Long AD Genome-wide analysis of a long-term evolution experiment with Drosophila. Nature. 2010:467(7315):587-90.

27. Schlotterer C, Kofler R, Versace E, Tobler R, Franssen SU. Combining experimental evolution with next-generation sequencing: a powerful tool to study adaptation from standing genetic variation. Heredity (Edinb). 2015:114(5):431-40.

28. Turner TL, Stewart AD, Fields AT, Rice WR, Tarone AM. Population-based resequencing of experimentally evolved populations reveals the genetic basis of body size variation in Drosophila melanogaster. PLoS Genet. 2011; 7(3):e1001336.

29. Remolina SC, Chang PL, Leips J, Nuzhdin SV, Hughes KA. Genomic basis of aging and life-history evolution in Drosophila melanogaster. Evolution. 2012 66(11):3390-403.

30. Jha AR, Miles CM, Lippert NR, Brown CD, White KP, Kreitman M. WholeGenome Resequencing of Experimental Populations Reveals Polygenic Basis of Egg-Size Variation in Drosophila melanogaster. Mol Biol Evol. 2015:32(10):2616-32

31. Cooper TF, Rozen DE, Lenski RE. Parallel changes in gene expression after 20,000 generations of evolution in Escherichia coli. Proc Natl Acad Sci U S A. 2003;100(3):1072-7.

32. Pelosi L, Kuhn L, Guetta D, Garin J, Geiselmann J, Lenski RE, et al. Parallel changes in global protein profiles during long-term experimental evolution in Escherichia coli. Genetics. 2006;173(4):1851-69.

33. Woods R, Schneider D, Winkworth CL, Riley MA, Lenski RE. Tests of parallel molecular evolution in a long-term experiment with Escherichia coli. Proc Natl Acad Sci U S A. 2006;103(24):9107-12. 
34. Barrick JE, Yu DS, Yoon SH, Jeong H, Oh TK, Schneider D, et al. Genome evolution and adaptation in a long-term experiment with Escherichia coli. Nature. 2009;461(7268):1243-7.

35. Gerstein AC, Lo DS, Otto SP. Parallel genetic changes and nonparallel geneenvironment interactions characterize the evolution of drug resistance in yeast. Genetics. 2012;192(1):241-52.

36. Chandler CH. Parallel Genome-Wide Fixation of Ancestral Alleles in Partially Outcrossing Experimental Populations of Caenorhabditis elegans. G3 (Bethesda). 2014;4(9):1657-65.

37. Gibbs AG. Water balance in desert Drosophila: lessons from non-charismatic microfauna. Comp Biochem Physiol A Mol Integr Physiol. 2002;133(3):781-9.

38. Gibbs AG, Matzkin LM. Evolution of water balance in the genus Drosophila. J Exp Biol. 2001;204(Pt 13):2331-8

39. Matzkin LM, Watts TD, Markow TA. Desiccation resistance in four Drosophila species: sex and population effects. Fly (Austin). 2007;1(5):268-73.

40. Gibbs AG, Fukuzato F, Matzkin LM. Evolution of water conservation mechanisms in Drosophila. J Exp Biol. 2003:206(Pt 7):1183-92.

41. Djawdan M, Chippindale AK, Rose MR, Bradley TJ. Metabolic reserves and evolved stress resistance in Drosophila melanogaster. Physiol Zool. 1998; 71(5):584-94.

42. Matzkin LM, Markow TA. Transcriptional regulation of metabolism associated with the increased desiccation resistance of the cactophilic Drosophila mojavensis. Genetics. 2009:182(4):1279-88.

43. Rajpurohit S, Oliveira CC, Etges WJ, Gibbs AG. Functional genomic and phenotypic responses to desiccation in natural populations of a desert drosophilid. Mol Ecol. 2013;22(10):2698-715.

44. Aggarwal DD, Rashkovetsky E, Michalak P, Cohen I, Ronin Y, Zhou D, et al. Experimental evolution of recombination and crossover interference in Drosophila caused by directional selection for stress-related traits. BMC Biol. 2015:13(1):101.

45. Pool JE, Corbett-Detig RB, Sugino RP, Stevens KA, Cardeno CM, Crepeau MW, et al. Population Genomics of sub-saharan Drosophila melanogaster: African diversity and non-African admixture. PLoS Genet. 2012;8(12):e1003080.

46. Syed ZA, Härd T, Uv A, van Dijk-Härd IF. A potential role for Drosophila mucins in development and physiology. PLoS One. 2008;3(8):e3041.

47. Belay AT, Scheiner R, So AKC, Douglas SJ, Chakaborty-Chatterjee M, Levine JD, et al. The foraging gene of Drosophila melanogaster: spatial-expression analysis and sucrose responsiveness. J Comp Neurol. 2007:504(5):570-82.

48. Johnson E, Sherry T, Ringo J, Dowse H. Modulation of the cardiac pacemaker of Drosophila: cellular mechanisms. J Comp Physiol B Biochem Syst Environ Physiol. 2002;172(3):227-36

49. Sokolowski MB. Drosophila: genetics meets behaviour, vol. 2. 2001. p. 879-90.

50. Baker BS, Taylor BJ, Hall JC. Are complex behaviors specified by dedicated regulatory genes? Reasoning from Drosophila, vol. 105. 2001. p. 13-24

51. Zhang S, Dailey GM, Kwan E, Glasheen BM, Sroga GE, Page-McCaw A. An MMP liberates the Ninjurin A ectodomain to signal a loss of cell adhesion. Genes Dev. 2006;20(14):1899-910.

52. FlyBase C, Swiss-Prot Project M, InterPro Project M. Gene Ontology annotation in FlyBase through association of InterPro records with GO terms. 2004. (The FlyBase Consortium)

53. Chang CB, Hemmati-Brivanlou A. Cell fate determination in embryonic ectoderm. J Neurobiol. 1998;36(2):128-51.

54. Orozco-terWengel P, Kapun M, Nolte V, Kofler R, Flatt T, Schlotterer C. Adaptation of Drosophila to a novel laboratory environment reveals temporally heterogeneous trajectories of selected alleles. Mol Ecol. 2012;21(20):4931-41.

55. Turner TL, Miller PM. Investigating Natural Variation in Drosophila Courtship Song by the Evolve and Resequence Approach. Genetics. 2012;191(2):633-42

56. Franssen SU, Nolte $V$, Tobler R, Schlotterer C. Patterns of linkage disequilibrium and long range hitchhiking in evolving experimental Drosophila melanogaster populations. Mol Biol Evol. 2015;32(2):495-509.

57. Bajpe PK, van der Knaap JA, Demmers JA, Bezstarosti K, Bassett A, van Beusekom HM, et al. Deubiquitylating enzyme UBP64 controls cell fate through stabilization of the transcriptional repressor tramtrack. Mol Cell Biol. 2008;28(5):1606-15.

58. Ribaya JP, Laski FA. Characterization of mule, a gene encoding a deubiquitinating enzyme required for ovary and testes germline development. In: Program and Abstracts 46th Annual Drosophila Research Conference, San Diego, CA, 2005. 2005. p. 550A.

59. Taillebourg E, Fauvarque M. The dUSP36 Ubiquitin Specific Protease is required for larval growth and moulting. In: Program and Abstracts 48th Annual Drosophila Research Conference, Philadelphia, PA, 2007. 2007. p. 854B.
60. Karouzou MV, Spyropoulos Y, Iconomidou VA, Cornman RS, Hamodrakas SJ, Willis $\mathrm{JH}$. Drosophila cuticular proteins with the R\&R Consensus: annotation and classification with a new tool for discriminating RR-1 and RR-2 sequences. Insect Biochem Mol Biol. 2007:37(8):754-60.

61. O'Mahony PJ, Oliver MJ. The involvement of ubiquitin in vegetative desiccation tolerance. Plant Mol Biol. 1999:41(5):657-67.

62. Adhikari BN, Wall DH, Adams BJ. Desiccation survival in an Antarctic nematode: molecular analysis using expressed sequenced tags. BMC Genomics. 2009;10:69.

63. Schilman PE, Lighton JR, Holway DA. Respiratory and cuticular water loss in insects with continuous gas exchange: comparison across five ant species. J Insect Physiol. 2005;51(12):1295-305.

64. Pavlidis P, Jensen JD, Stephan W, Stamatakis A. A critical assessment of storytelling: gene ontology categories and the importance of validating genomic scans. Mol Biol Evol. 2012;29(10):3237-48.

65. Zhou D, Udpa N, Gersten M, Visk DW, Bashir A, Xue J, et al. Experimental selection of hypoxia-tolerant Drosophila melanogaster. Proc Natl Acad Sci U S A. 2011:108(6):2349-54.

66. Jalvingh KM, Chang PL, Nuzhdin SV, Wertheim B. Genomic changes under rapid evolution: selection for parasitoid resistance. Proc Biol Sci. 2014; 281(1779):20132303.

67. Chevin LM, Hospital F. Selective sweep at a quantitative trait locus in the presence of background genetic variation. Genetics. 2008;180(3):1645-60.

68. Pavlidis P, Metzler D, Stephan W. Selective sweeps in multilocus models of quantitative traits. Genetics. 2012:192(1):225-39.

69. Tobler R, Franssen SU, Kofler R, Orozco-terWengel P, Nolte V, Hermisson J, et al. Massive Habitat-Specific Genomic Response in D. melanogaster Populations during Experimental Evolution in Hot and Cold Environments. Mol Biol Evol. 2014;31(2):364-75.

70. Hoffmann AA, Harshman LG. Desiccation and starvation resistance in Drosophila: patterns of variation at the species, population and intrapopulation levels. Heredity (Edinb). 1999;83(Pt 6):637-43.

71. Parkash R, Singh D, Lambhod C. Sex-specific differences in desiccation resistance and the use of energy metabolites as osmolytes in Drosophila melanogaster flies acclimated to dehydration stress. J Comp Physiol B. 2014; 184(2):193-204.

72. Aronesty E. Comparison of Sequencing Utility Programs. Open Bioinformatics J. 2013;7:1-8.

73. Li H, Durbin R. Fast and accurate short read alignment with Burrows-Wheeler transform. Bioinformatics. 2009;25(14):1754-60.

74. DePristo MA, Banks E, Poplin R, Garimella KV, Maguire JR, Hartl C, et al. A framework for variation discovery and genotyping using next-generation DNA sequencing data. Nat Genet. 2011:43(5):491-8.

75. Li H, Handsaker B, Wysoker A, Fennell T, Ruan J, Homer N, et al. The Sequence Alignment/Map format and SAMtools. Bioinformatics. 2009;25(16):2078-9.

76. Kofler R, Pandey RV, Schlotterer C. PoPoolation2: identifying differentiation between populations using sequencing of pooled DNA samples (Pool-Seq). Bioinformatics. 2011;27(24):3435-6.

77. Kofler R, Orozco-terWengel P, De Maio N, Pandey RV, Nolte V, Futschik A, et al. PoPoolation: a toolbox for population genetic analysis of next generation sequencing data from pooled individuals. PLoS One. 2011;6(1):e15925.

78. Kessner D, Novembre J. forqs: forward-in-time simulation of recombination, quantitative traits and selection. Bioinformatics. 2014;30(4):576-7.

79. Kofler R, Schlotterer C. Gowinda: unbiased analysis of gene set enrichment for genome-wide association studies. Bioinformatics. 2012;28(15):2084-5.

80. Mcdonald JH, Kreitman M. Adaptive Protein Evolution at the Adh Locus in Drosophila. Nature. 1991;351(6328):652-4.

81. Nei M, Gojobori T. Simple methods for estimating the numbers of synonymous and nonsynonymous nucleotide substitutions. Mol Biol Evol. 1986;3(5):418-26. 